\title{
Noise in Olive Mills, the Case of Jordan: Actual Measurements \& Reduction Proposition
}

\author{
Rizeq N. Hammad, May M. Hourani*, Firas M. Sharaf \\ Department of Architecture, Faculty of Engineering, Jordan University, Amman, Jordan
}

Email address:

rizeqhammad@yahoo.com (R. N. Hammad),may_hourani@yahoo.com (M. M. Hourani), f.sharaf@ju.edu.jo (F. M. Sharaf)

To cite this article:

Rizeq N. Hammad, May M. Hourani, Firas M. Sharaf. Noise in Olive Mills, the Case of Jordan: Actual Measurements \& Reduction Proposition. International Journal of Mechanical Engineering and Applications. Vol. 3, No. 3, 2015, pp. 46-49. doi: 10.11648/j.ijmea.20150303.13

\begin{abstract}
This study is concerned mainly with high noise produced by automated olive mills and its negative impact on human health. This study looks at what caused this problem such as the application of building codes concerning user exposure to noise and protective measures for workers. Long working period exposure to high noise, particularly mill workers, causes health and ear illness. Noise levels are measured in several olive mills in Jordan, recorded data was analyzed and findings indicated that noise level is higher than the maximum noise limits allowed by the Jordanian code. This study concludes design solutions and legislative procedures to improve noise control in olive mills.
\end{abstract}

Keywords: Olive Mills Design, High Noise Impact, Noise Reduction

\section{Introduction}

Olive oil is a basic product in the lives of Mediterranean people, both for nutrition and other related products. Olive oil is used as a raw material for many industries such as soap, cosmetics and medicines. The Mediterranean region is a main source for olive cultivation. Since the olive tree is considered a blessed tree in many religions, it is widely planted in the Middle Eastern region for hundreds of years.

In Jordan, there are approximately 20 million olive trees planted in 1.3 million acres, and almost $77 \%$ of olive trees are rain fed cultivated. The olive sector is a main contributor to the economy and national gross production in Jordan. The amount of annual income from the olive industry in Jordan is about 100 million Jordanian Dinars (JDs, about 150 million US dollars). Investment in the olive industry is increasing and the size of this investment reaches one billion JDs, this amount includes the value of planted land and industries. The olive industry in Jordan has social importance as sustenance for a large segment of farmers and other involved people [1].

A considerable change is taking place in olive mill design in Jordan because of the development in the olive industry in the last decade. More olive trees are planted and increased number of modern olive mills producing more oil and better quality. Modern automatic pressing machines have invaded the Jordanian market and replaced traditional olive oil extraction methods. The ratio of modern olive mills in Jordan is $93 \%$ while ratio of traditional olive mills is $7 \%$. There are about 124 olive oil mills in Jordan with a production capacity of approximately 352 tons/hour. These mills include 245 advanced production lines of high quality olive oil. ${ }^{1}$

\section{Architectural Design of Olive Oil Mills}

A typical shape of a modern olive oil mill is rectangular; a fore-entrance leads to a main hall where olive oil is filled into cans under supervision of olive holders. A rear entrance is used to bring in olives inside the mill. The area used for pressing olives is not open to public, but visually connected to the entrance hall, so that everyone can observe olive oil production processes. Entrance hall is used for filling oil into metal containers and for loading and unloading bottled and metal containers. "Fig. 1" shows a typical layout of a modern olive oil mill in Jordan.

Employees of the mill are responsible for monitoring, accounting and weighing; therefore the employees' offices are located at the front of mill to allow monitoring the processes of olive pressing and filling oil in steel containers. These functions are located within one space in the mill of about 4 meters height and roofed by concrete slab, or steel roof with frames covered with aluminum panels. Olives are put in bags

1 Royal Scientific Society, Environmental Record Centre, Clear Production Unit. Amman - Jordan 
in a storage area for few days awaiting pressing. The processes of producing olive oil in a modern automated mill includes washing, crushing, grinding, centrifugal oil extraction and dumping remains (peat) out of the production line "Fig. 2" The size of olive oil mill depends on the number of production lines which ranges from one to three lines. The length of the mill ranges between $15-30 \mathrm{~m}$ and width between $10-15 \mathrm{~m}$. "Fig. 3": A, B, C show the interior of a modern olive oil mill in Jordan. Interior finishing of the mill is basic, such as a painted cement plastered surface and in some cases the concrete structure is left unfinished. Perhaps, this is because mills are used for three months only every year and close until next olive season.

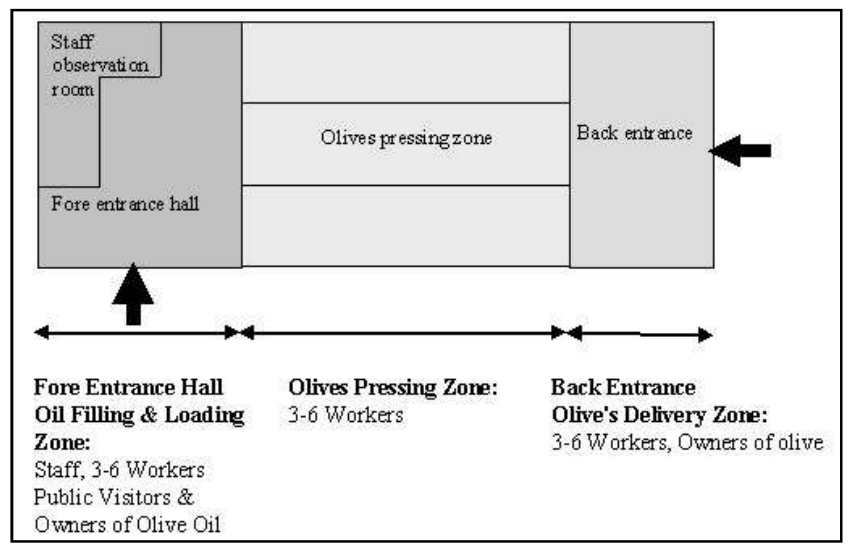

Figure 1. Typical layout of modern olive oil mill.

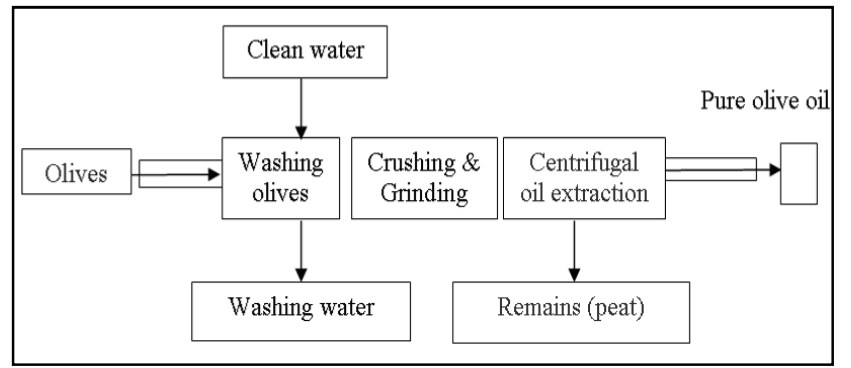

Figure 2. Olive oil production processes in a modern mill.

\section{Noise Problem in Modern Olive Oil Mill}

Introducing modern olive oil pressing machinery into mills has increased the production volume of olive oil while maintaining good quality and reducing human effort and time to extract olive oil.

However, modern machines have caused negative consequences for environment and user health. High noise levels rising to risk limits in olive oil mills is considered the most serious negative impact on workers, and other users such as visitors. In addition to the other problems of modern oil mills that are not the subject of this study, such as the greater consumed amount of water compared to traditional methods and producing residues which negatively affect environment and people.
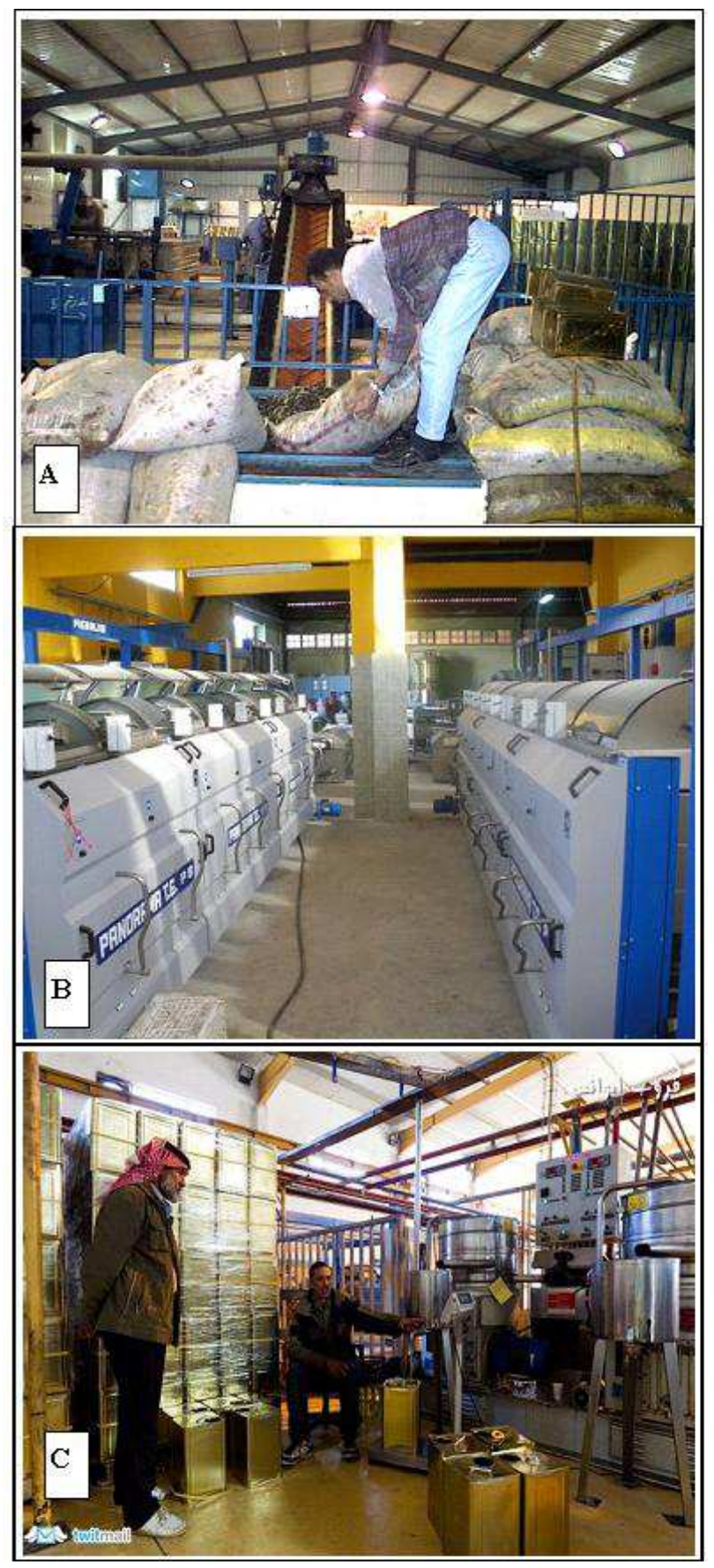

Figure 3. Olive oil mill. A: Back-house: loading olives in the mill. B: pressing machines in the mill. C: Fore-house: filling oil into cans.

The International Olive Council guidelines [2] recommends reducing harmful noise impact of oil mill by separating the grinding and crushing machines from the olive delivery area at rear entrance and also from the front area at the entrance where olive oil is collected and filled in containers ready for transportation. Workers in olive oil mills need to wear suitable ear protection at all time to minimize negative effects of high noise levels.

Although modern olive mills produce high continuous noise, little research has been conducted on this subject, which this paper seeks to address.

The duration of exposure, or daily dose (DOSE), to noise 
level $95 \mathrm{~dB}(\mathrm{~A})$ allowed by The International standard is 3 hours a day per five days a week [3]; and according to American standards is 4 hours a day per working week [4].

\section{Previous Studies}

A common conclusion in previous studies $[5,6,7,8]$ is that noise levels are generally high in olive oil mills and create risk for workers, who generally work more than 8 hours a day for about two months during olive season. In a study which investigates noise levels in several olive oil mills in Italy [9], a country known for its wide spread of olive mills and large production of olive oil, noise levels were recorded in different locations inside each mill to find out the average noise level.

Conclusions of this study in Italy are:

1. Noise levels in the study mills are generally high and exceed maximum noise limits of international standards.

2. The main source of noise is from the centrifugal extractor, washing machines and steel hammer crushing machine.

3. The size of mill's building has a significant influence on noise levels because of reverberant noise.

4. The Average recorded noise level was $93.7 \mathrm{~dB}(\mathrm{~A})$ at some mills which exceeded the allowed exposure limits that should not last more than 1.1 to 1.7 hours per day at maximum.

The study of olive oil mills in Italy suggested noise treatments such as sound absorption panels fixed on walls to limit reverberation. Results from this study are presented in "Fig. 4".

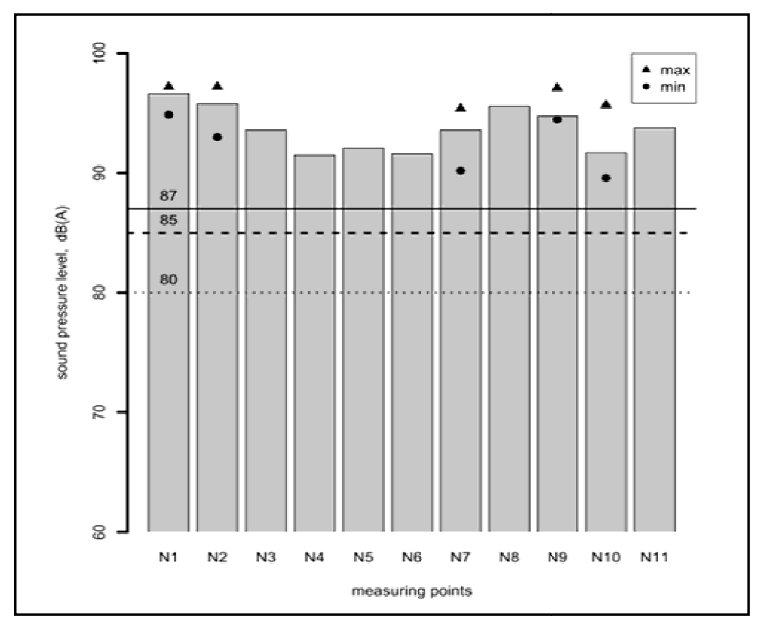

Figure 4. Results of sound pressure levels from oil mills' study in Italy [9].

\section{Methodology and Procedure}

Field visits to olive oil mills in Jordan were conducted to record noise levels. The surveyed mills mostly use the same equipment and olive pressing methods; the main difference between mills was the number of production lines which ranges from one to four production lines.

Noise of mill equipment is generally constant; therefore this noise could be stated by the weight $\mathrm{AdB}(\mathrm{A})$ which is certified in Jordanian and International Standards.

A Sound Pressure Level Meter (B \& K type 2215) was used to measure noise level in the surveyed mills of a frequency range $63-8000 \mathrm{~Hz}$. A One Third Octave Band Filter was used to assess different frequencies especially for less than $500 \mathrm{~Hz}$.

Filed visits to the mills were carried out at different times in the week, as mills operate 24 hours a day in olive harvesting season. Time and date of measurement were recorded. Noise level values were recorded at the front area of the mill where many people such as workers and buyers use to collect and buy olive oil. Selection of the front area of the mill to conduct noise measurements is because of the larger number of people use this area such as workers, staff, olive owners and oil buyers. Other areas of the mill are used by limited number of people such as workers and mill owners who are responsible for supervising the maintaining of equipment and production lines.

\section{Results and Discussion}

Recorded sound levels are high especially at low frequencies which vary from 85 to $95 \mathrm{~dB}(\mathrm{~A})$ "Fig. 5".

A comparison between measured noise level values in olive oil mills in Jordan "Fig. 5" and those in Italy "Fig. 4" show similarities in the recorded values.

Workers in the surveyed olive oil mills in Jordan work for at least 8 hours a day in high noise level conditions in the mills; some workers even work for two shifts of 16 hours a day.

Comparing the measured values in the study mills in Jordan to international standards show that workers in olive mills are exposed to high sound levels for long hours which can cause hearing problems, such as partial or total deafness and physical and psychological risks according to The World Health Organization (WHO) [10]. It was noted that there was no ears protective tools or work clothes for staff and workers in the surveyed mills. Owners of mills often show little interest to protective measures from high noise in the mill. Architectural design of surveyed olive mills in Jordan show little consideration to protect users from high noise. The pressing machinery area is completely open towards the public and workers. Quality of buildings and interior finishing in the surveyed mills is generally basic just to provide a roof and protection from sun and climate conditions.

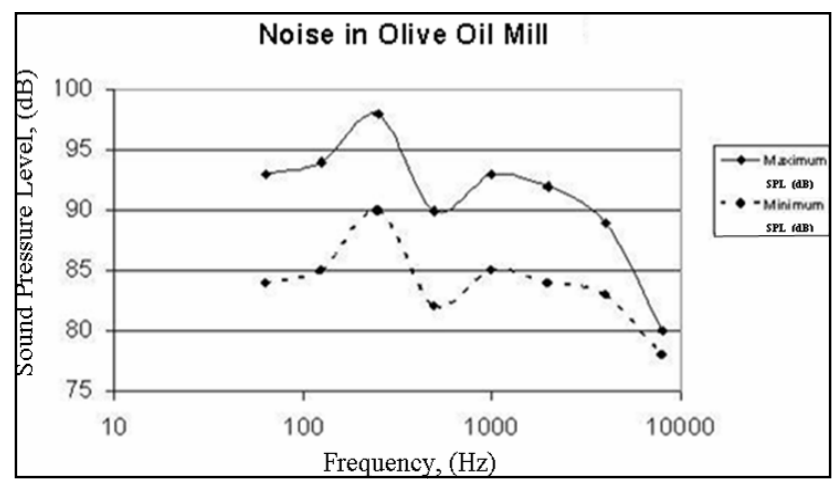

Figure 5. Noise level Measurements in olive oil mills-Jordan (85-95) dB(A). 
This study would present some proposals to improve the acoustical conditions in olive oil mills. Proper architectural design of olive oil mills can help to separate users from the exposure to high noise levels produced by machines. It is also possible to organize the working hours of the staff to reduce the duration of noise exposure; aiming to meet the allowed duration of noise exposure related to a measured noise level (Dose). The International standards limit the exposure to $85 \mathrm{~dB}(\mathrm{~A})$ noise level for a maximum duration of 8 hours a day during five working days a week. Lack of awareness about serious health threats caused by high noise levels is noted in the surveyed mills in Jordan. Building legislation should enforce safety codes on mills to force owners to implement safety measures and subject disobedient to legal and financial penalties. Raising awareness of owners about noise risks in mills is necessary but needs time and effort.

This research suggests noise reduction in olive mills by using practical and low cost solutions to limit possible health risks of users caused by long exposure to noise in mills. "Fig. 6 " shows a vertical section of a proposed wall that can separate the pressing machines area from the area used by workers and customers in mills. The wall has large glazed area which helps keeping visual connectivity between the pressing machines and olive owners to monitor and maintain quality control during olive oil extracting process. The separating wall can reduce noise level by at least $25 \mathrm{~dB}(\mathrm{~A})$ and lowering noise level to $70 \mathrm{~dB}(\mathrm{~A})$. This solution allows 8 hour exposure to noise a day without causing health implications to users.

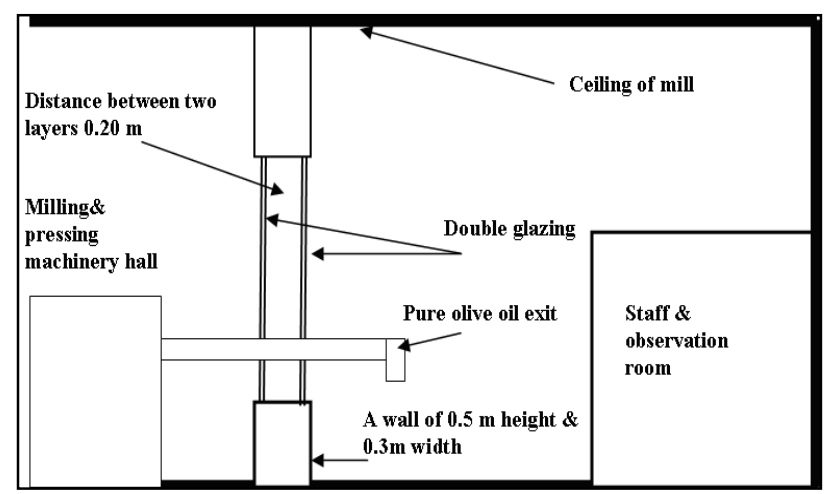

Figure 6. Proposed vertical section in olive oil mill.

\section{Conclusion}

1. Noise levels were measured in several olive oil mills in Jordan, the recorded noise levels and exposure duration exceeded maximum amounts as allowed by Jordanian code. International standards limit maximum exposure duration to $85 \mathrm{~dB}(\mathrm{~A})$ for 8 hours a day five working days a week. Workers in the study mills are found to be exposed to noise level of (85-95) $\mathrm{dB}(\mathrm{A})$ for a duration reaching up to three times than the allowed duration.

2. Noise levels recorded in the study mills in Jordan came close to noise levels recorded in the case of olive oil mills in Italy. This means that high noise levels in olive mills is a problem in many countries that needs to be addressed.

3. This study suggests reducing olive oil mills' noise through isolation of the equipment that produces noise from users, while keeping direct visual observation of olive oil production lines.

\section{References}

[1] A. Khadair, Cleaner Production in Olive Oil Industry in Jordan, In The 2nd Arab Clear Production Workshop. Amman: Jordan. August 28-30, 2007.

[2] International Olive Council, Quality Management Guide for the Olive Oil Industry: Olive Oil Mills. Madrid, Spain, 2006.

[3] International Organization for Standardization, ISO 1999: 1990 Acoustics- determination of occupational noise exposure and estimation of noise-induced hearing impairment. Geneva: Switzerland, 2006.

[4] Occupational Safety and Health Administration, Occupational noise exposure: (OSHA 1910.95 App). Department of Labor, USA

[5] U. Landstrm, E. Akerlund, A. Kjellberg, M. Tesarz, "Exposure Levels, Tonal Components, and Noise Annoyance in Working Environments," Environment International journal 1995; vol. 21 no 3: 265-275

[6] PJ. Middendorf, "Surveillance of Occupational Noise Exposures Using OSHA's Integrated Management Information System", American Journal of Industrial Medicine 2004; vol. 46, pp. 492-504.

[7] GV. Prasanna Kumar, KN. Dewangan, A. Sarkar, "Noise exposure in oil mills", Indian J. Occup. Environ. Med, 2008; 12 no1, pp. 23-28.

[8] V. Panaro, S. Pascuzzi, F. Santoro, "Analysis of exposition to noise in olive oil production sector: measurements during mechacnical harvesting and oil extraction [Apulia]", Journal of Agricultural Engineering 2004, Vol 35 issue2

[9] G. Manetto, E. Cerruto, G. Emma, Noise operator exposure in olive oil mills, In: International Conference RAGUSA SHWA 2012. Ragusa - Italy. 2012. "Safety Health and Welfare in Agriculture and in Agro- food Systems".

[10] The World Health Organization (WHO), Occupational Health, chapter 21: Selected occupational risk factors. 